\title{
Intrinsic profile analysis of intracranial cerebrospinal fluid
}

\author{
Alain Lebrett,*, Yukiko Kenmochi ${ }^{\mathrm{b}}$, Toru Tamaki ${ }^{\mathrm{c}}$ \\ ${ }^{a}$ Normandie Université, ENSICAEN, Caen, France \\ ${ }^{b}$ Université Paris-Est, LIGM, CNRS, Marne-la-Vallée, France \\ ${ }^{c}$ Hiroshima University, Hiroshima, Japan
}

\begin{abstract}
Purpose: We aim at studying intrinsic structures of the intracranial subarachnoid space.

Material and methods: Magnetic resonance images were obtained using the SPACE sequence, and the segmentation of the superior intracranial subarachnoid space was performed using geometrical features and a topological assumption of the shapes. Given such segmentation results, we present a method based on a geodesic propagation technique, which allows us to make an intrinsic profile of the space. Intrinsic profiles are then analyzed qualitatively and quantitatively, in particular for classification into healthy and pathological cases based on their intrinsic bilateral asymmetry and histogram moments.

Results: The proposed method was applied to a clinical dataset of 15 subjects, of which 7 were healthy volunteers and 8 were hydrocephalus patients. The intracranial cerebrospinal fluid is not (intrinsically) bilaterally asymmetric for healthy volunteers, while hydrocephalus would cause asymmetry. We also observed that the results of a two-class classification (healthy or not) based on histogram moments were suitable; sensitivity, specificity and precision are all $100 \%$.

Conclusions: The effectiveness of the proposed method of intrinsic profiling analyses is shown by preliminary experiments on healthy adults and hydrocephalus patients.
\end{abstract}

Keywords: Intracranial subarachnoid space, geodesic propagation, intrinsic shape analysis, classification

\section{Introduction}

The central nervous system is surrounded by cerebrospinal fluid that is contained within the cerebral ventricles and subarachnoid space $[1,2]$. The observation of cerebrospinal fluid plays a valuable role in the clinical diagnosis of brain disorders such as hydrocephalus and Alzheimer's disease [2, 3, 4]. One example is hydrocephalus, which usually results from obstructed fluid outflows in the ventricles or subarachnoid space that leads to an alteration in fluid volumes [3]. Therefore, confirmation of changes to fluid volumes can help in its diagnosis [5]. The fluid distribution and the network of intracranial subarachnoid space were also explored using the volumetric relief map [6], and their bilateral symmetry and asymmetry were observed for healthy adults and patients, respectively.

\footnotetext{
${ }^{*}$ Corresponding author.

URL: alain.lebret@ensicaen.fr (Alain Lebret), yukiko.kenmochi@esiee.fr (Yukiko Kenmochi), tamaki@hiroshima-u.ac.jp (Toru Tamaki)
} 
In this article, we extend this study and expound a method based on a geodesic propagation technique to obtain not only global information but also more local shape information. Geodesic distances have been lately used in many shape processing applications, such as shape classification [7], recognition [8], retrieval [9], and symmetry detection [10], especially for articulated shapes, thanks to the robustness to shape variations by non-rigid deformations. Indeed, there exist shape variations of the human body by non-rigid deformations due to individual variation and asymmetry [11]. Here, we present a simple method based on geodesic distances to study intrinsic structures of the intracranial subarachnoid space.

Concretely, we observe geodesic propagation from specific fluid sources, which correspond to the ventricular space outputs, which are symmetrically located nearly at the brain center and can be detected due to the specific shapes. To analyze such propagation, we propose to use a geodesic propagation histogram, which allows us to make an intrinsic profile of such space. Intrinsic profiles are then analyzed qualitatively with the help of two visualization tools: 3D volume rendering and 2D volumetric relief map [6]. They allow us to understand how the geodesic propagation is made in the intracranial subarachnoid space, and thus to detect key distances where the manner of propagation evolution changes. For the quantitative analysis, a simple but effective method for detecting the bilateral intrinsic asymmetry using a geodesic histogram correlation is proposed. As a complement, classification into healthy and pathological cases based on histogram moments is also made.

The effectiveness of the proposed method is shown by preliminary experiments on healthy adults and hydrocephalus patients.

\section{Material and methods}

\subsection{MRI data and pre-segmentation}

Magnetic resonance images were obtained using the SPACE sequence described in [12] and had an isotropic voxel resolution of $1 \mathrm{~mm}$. The segmentation of the superior intracranial subarachnoid space was performed based on the method given in [5], which guarantees the connectivity of the segmented volume. More precisely, the cerebrospinal fluid was first pre-segmented using geometrical features and a topological assumption of the shapes [5] and then the intracranial subarachnoid space was extracted [5]. Given such a fluid volume, we first present a geodesic propagation technique for generating its intrinsic profile, and then propose several methods for analysing those intrinsic profiles.

\subsection{Geodesic propagation in the cerebrospinal fluid}

Regarding image voxel centers as $3 \mathrm{D}$ regular grid points $\mathbb{Z}^{3}$, each voxel at $\mathbf{x} \in \mathbb{Z}^{3}$ is represented by the closed grid cube $\Omega(\mathbf{x})=\mathbf{x}+\left[-\frac{1}{2}, \frac{1}{2}\right]^{3} \subset \mathbb{R}^{3}$. Given a pre-segmented voxel set $V \subset \mathbb{Z}^{3}$, let us consider the union of voxels whose centers $\mathbf{x}$ are in $V$, i.e., $\Upsilon(V)=\cup_{\mathbf{x} \in V} \Omega(\mathbf{x}) \subset \mathbb{R}^{3}$.

Now we define a (piecewise) smooth curve $\gamma$ in the region $\Upsilon(V)$ with a parameter $t \in[0,1]$ such that $\gamma(0)=\mathbf{u}$ and $\gamma(1)=\mathbf{v}$. The length of $\gamma$ is then defined as

$$
L(\gamma)=\int_{0}^{1}\left\|\gamma^{\prime}(t)\right\| d t
$$

where $\gamma^{\prime}(t)$ is the derivative of $\gamma$. 
Let $\Pi(\mathbf{u}, \mathbf{v})$ be the set of all the curves in $\Upsilon(V)$ between $\mathbf{u}$ and $\mathbf{v}$. Then, the geodesic distance between $\mathbf{u}$ and $\mathbf{v}$ in $V$ is defined by

$$
d_{V}(\mathbf{u}, \mathbf{v})=\min _{\gamma \in \Pi(\mathbf{u}, \mathbf{v})} L(\gamma) .
$$

The curve that has the minimum length is called the "shortest path."

Let $S$ be the set of fluid sources located in the voxel set $V$. Let us now consider for each $\mathbf{v} \in V$ the geodesic distance $d_{V}(\mathbf{s}, \mathbf{v})$ from a source $\mathbf{s} \in S$. Given a fluid source set $S$, we can then define the minimum geodesic distance

$$
\operatorname{Dis}_{V}^{S}(\mathbf{v})=\min _{\mathbf{s} \in S} d_{V}(\mathbf{s}, \mathbf{v})
$$

for each voxel $\mathbf{v} \in V$. The map $D i s_{V}^{S}$ is called a geodesic distance map of $V$ for $S$. To compute this map, we apply the fast marching method based on front propagation presented in [13], which is an efficient one-pass algorithm similar to the Dijkstra algorithm [14].

One of the critical issues in the analysis of fluid propagation pathways using such geodesic propagation technique is to set the fluid source $S$. We set our source $S$ at the outputs of the ventricular space, which are symmetrically located nearly at the brain center and, in practice, detectable due to the specific shapes. This source location is important to obtain the intrinsic profile that preserves the shape symmetry of a fluid volume $V$ (if there is). Concerning the detection of $S$, a practical technique is explained, for example, in the next experimental section. Such fluid sources are called seeds for the geodesic propagation procedure.

\subsection{Intrinsic profiling of intracranial cerebrospinal fluid}

Once a geodesic distance map $D i s_{V}^{S}$ is calculated for a fluid volume $V$ from a given source set $S$, the histogram is defined by

$$
h_{D i s_{V}^{S}}(d)=\left|\left\{\mathbf{v} \in V:\left[D i s_{V}^{S}(\mathbf{v})\right]=d\right\}\right|
$$

for non-negative integers $d$ in the range of $\operatorname{Dis}_{V}^{S}$ where $|\cdot|$ is the set cardinality and [.] is the round function, such that the bin interval is simply set 1 in this article. The histogram is then analyzed for intrinsic profiling of the intracranial cerebrospinal fluid $V$. In other words, geodesic propagation histograms allow us to extract intrinsic structural information of $V$.

\subsection{Geodesic propagation visualization}

To analyze qualitatively geodesic propagation, the simplest idea is to visualize such a geodesic distance map $D i s_{V}^{S}$ using the technique of volume rendering with colors that correspond to distances. However, as the shape of the intracranial cerebrospinal fluid $V$ is complex, the analysis is not straightforward; additional manipulations such as viewpoint changes, partial object visualization, and cross-section visualization usually behoove, all of which is time-consuming.

To overcome those disadvantages, we have proposed to represent the geodesic distance map $D i S_{V}^{S}$ alternatively by a sequence of two-dimensional images, called volumetric relief maps [6]. Given a volume, its volumetric relief map is generated as follows. First, we project each voxel onto the hemisphere enclosing the volume, and then onto a plane. Each pixel of a volumetric relief map corresponds to the number of voxels projected in it. The key point to note is that the total fluid volume is preserved following these two projections, and thus in the volumetric relief 
map. The geodesic distances $\operatorname{Dis}_{V}^{S}(\mathbf{v})$ for all $\mathbf{v} \in V$ are thresholded at $d$ to obtain a series of subsets of $V$,

$$
V_{d}=\left\{\mathbf{v} \in V:\left[\operatorname{Dis}_{V}^{S}(\mathbf{v})\right] \leq d\right\},
$$

where $d=0,1, \ldots$, for which we generate a series of volumetric relief maps that can be viewed as an animation. This tool allows us to visualize in real time the voxels whose distances are observed as key ones in the histogram.

It should be mentioned that volumetric relief maps are generated for both left and right hemispheres in this article, while only the upper hemisphere was considered in [6], so that we can also observe the inferior intracranial subarachnoid space. In addition, we have adapted the seed setting for geodesic propagation, which is different from those proposed in [6], with the aim of this article.

\subsection{Bilateral intrinsic asymmetry detection}

To analyze quantitatively geodesic propagation histograms, we first consider the bilateral asymmetry of $V$, which was already observed via volumetric relief maps in pathological cases in the previous work [6]. Here we present a simple method using geodesic propagation histograms, which would be robust to shape variations by non-rigid deformations due to individual variation and asymmetry [11].

A volume $V$ is said to be (extrinsically) symmetric if there exists an isometric map $T: V \rightarrow V$ such that

$$
d_{\mathbb{R}^{3}}(\mathbf{u}, \mathbf{v})=d_{\mathbb{R}^{3}}(T(\mathbf{u}), T(\mathbf{v}))
$$

for any pair $\mathbf{u}, \mathbf{v} \in V$, where $d_{\mathbb{R}^{3}}$ is the Euclidean distance. In this article, $T$ is supposed to be the reflection with respect to the midsagittal plane from the anatomical reason. In other words, $\mathbf{u}=T(\mathbf{u})$ if $\mathbf{u}$ is on the midsagittal plane for such $T$. This particular symmetry is called the bilateral symmetry.

The main idea of the proposed method for detecting bilateral asymmetry of $V$ is based on the fact that geodesic propagation of left and right hemispherical parts of $V$ from the fluid sources positioned in a bilaterally balanced way, which was previously explained, are the same if $V$ is bilaterally symmetric. In other words, if $V$ is bilaterally symmetric, the geodesic propagation histograms of left and right hemispherical parts must be similar.

Let $h_{l}$ and $h_{r}$ be the two geodesic propagation histograms for left and right hemispherical parts. The similarity between $h_{l}$ and $h_{r}$ is computed by their correlation

$$
C\left(h_{l}, h_{r}\right)=\frac{\sum_{t}\left(h_{l}(t)-\overline{h_{l}}\right)\left(h_{r}(t)-\overline{h_{r}}\right)}{\sqrt{\sum_{t}\left(h_{l}(t)-\overline{h_{l}}\right)^{2} \sum_{t}\left(h_{r}(t)-\overline{h_{r}}\right)^{2}}}
$$

where $\overline{h_{*}}$ is the mean of $h_{*}(t)$ for all $t$.

In fact this similarity measure detects the intrinsic asymmetry, whose concept is wider than the classical extrinsic one defined in (2); $V$ is said to be intrinsically symmetric if there exists a homeomorphism $T: V \rightarrow V$ such that

$$
d_{V}(\mathbf{u}, \mathbf{v})=d_{V}(T(\mathbf{u}), T(\mathbf{v}))
$$

for any pair $\mathbf{u}, \mathbf{v} \in V$ [10]. This implies that the proposed similarity measure would provide robust results even though $V$ is not perfectly bilaterally (extrinsically) symmetric, as far as the metric structures of the right and left parts of $V$ are not significantly different. 
Another advantage of this method is that results are less affected by image digitization effect. A naive method for detecting extrinsical asymmetry may require an image reflection operation with respect to the midsagittal plane, which is generally not orthogonal to one of the image axes. As such an operation related to an image rotation is known to generate geometric and topological alterations in the transformed image, compared to the initial one [15], it should be avoided if possible. The proposed method indeed does not require such an image registration step.

\subsection{Feature vectors and intrinsic profile classification}

To analyze quantitatively geodesic propagation histograms, we also consider the shape of each histogram, which can be represented by the moments of the distribution of the mean geodesic distance [16]. In this article, after normalization of the histogram $h$, we use the first moment, the mean $m_{1}(h)$, the second central moment, the variance $m_{2}(h)$, the third central moment $m_{3}(h)$ that corresponds to the skewness, and the fourth moment $m_{4}(h)$ that corresponds to the kurtosis. Therefore, each histogram $h$ can be represented by a 4-dimensional feature vector $\mathbf{m}(h)=\left(m_{1}(h), m_{2}(h), m_{3}(h), m_{4}(h)\right)$.

To extract more partially detailed shape information of the histogram, we divide the spherical shape of the intracranial subarachnoid space into four equal parts (left-top, left-bottom, right-top, right-bottom) after the histogram computation. In other words, we divide the fluid region $V$ into the four distinct parts such that $V=V^{l t} \cup V^{l b} \cup V^{r t} \cup V^{r b}$. We then consider the four geodesic propagation histograms for those four parts, $h^{l t}, h^{l b}, h^{r t}$ and $h^{r b}$, each of which is defined by (1) replacing $V$ with $V^{*}$. Each histogram $h^{*}$ finally provides the four moments $\mathbf{m}\left(h^{*}\right)=\left(m_{1}\left(h^{*}\right), m_{2}\left(h^{*}\right), m_{3}\left(h^{*}\right), m_{4}\left(h^{*}\right)\right)$. Thus, with this subdivision, the original histogram can be characterized by a 16-dimensional feature vector $\left(\mathbf{m}\left(h^{l t}\right), \mathbf{m}\left(h^{l b}\right), \mathbf{m}\left(h^{r t}\right), \mathbf{m}\left(h^{r b}\right)\right)$.

Those 16-dimensional feature vectors are used to analyze data. More precisely, we make a model that classifies each input into either healthy or pathological case using support vector machine [17].

\section{Experimental results and discussion}

The proposed method was applied to a clinical dataset of 15 subjects. Of these, 7 were healthy volunteers (median age: 57 years; range: $25-84$ years) and 8 were hydrocephalus patients (median age: 58 years; range: $23-82$ years), where two of them are accompanied by further image acquisition post-surgery. Informed consent was obtained from all the subjects and studies using these data were approved by the institutional review board.

\subsection{Geodesic propagation}

An example of the geodesic distance map of intracranial cerebrospinal fluid for healthy adults diffused from three seeds located at the outputs of the ventricular space is shown in three dimensions with colors in Fig. 1. The figure indicates that the geodesic propagation seems bilaterally symmetric and that the furthest points reach the superior frontal gyri.

As a complement, volumetric relief map sequences for the right and left hemispheres are generated and allowed the monitoring of the fluid volume evolution within the entire subarachnoid space and its symmetry. Here only the right one is shown in Fig. 2. From $A$ to $C$, we see the flooding of brain cisterns that surround the cerebellum and the brainstem as of spaces under the lateral cerebral fissure. From $D$ to $F$, the superior intracranial subarachnoid space is progressively filled from lateral cerebral fissure to the anterior part of the longitudinal fissure. 

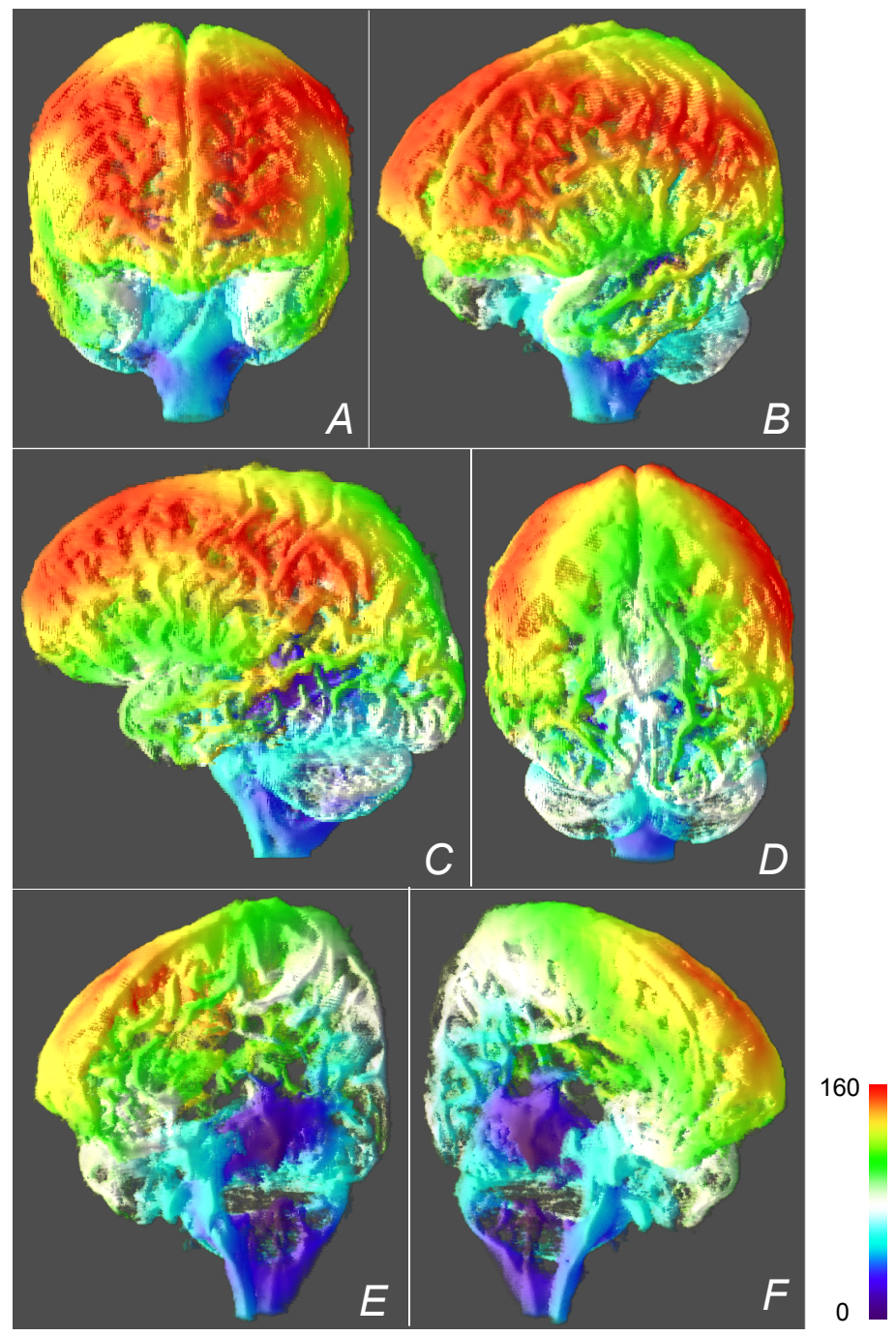

Figure 1: An example of the geodesic distance map of intracranial cerebrospinal fluid for healthy adults, propagated from three seeds located at the outputs of the ventricular space. The whole part is viewed from the front (A), left-front (B), left (C) and back (D), while the right and left insides are viewed separately (in (E) and (F), respectively) from different frontal viewpoints. 


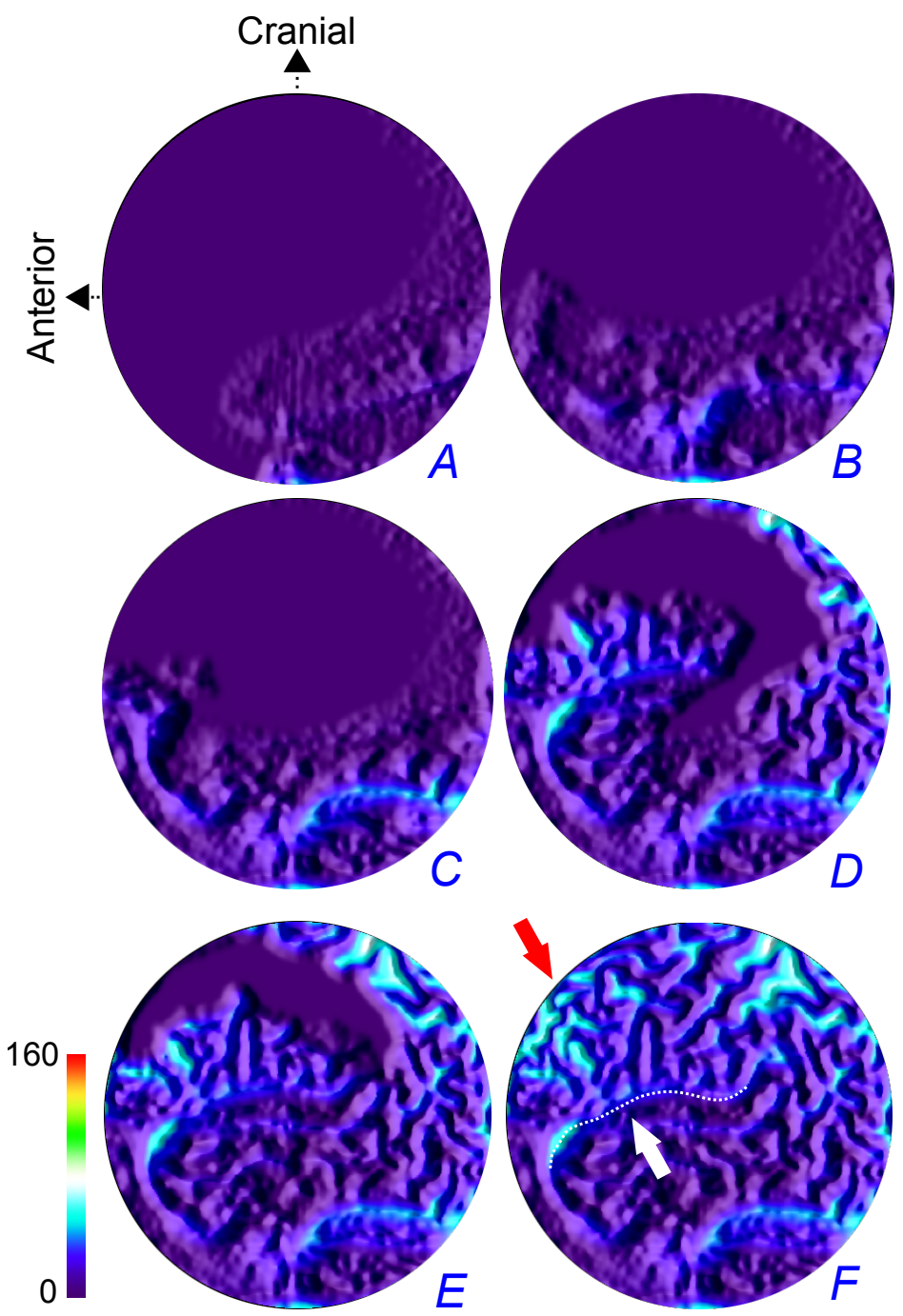

Figure 2: Extracts from a colored volumetric relief map sequence illustrating the geodesic distance map for the right hemisphere in Fig. 1 (see the attached mp4 file, video1, for the complete animation). The sequence begins at threshold distance $d=0$ and ends at $d=157$, and the maps were extracted at $d=29(A), 49(B), 68(C), 114(D), 126(E)$ and 157 $(F)$. The red arrow indicates the longitudinal cerebral fissure and the white one the lateral cerebral fissure. 


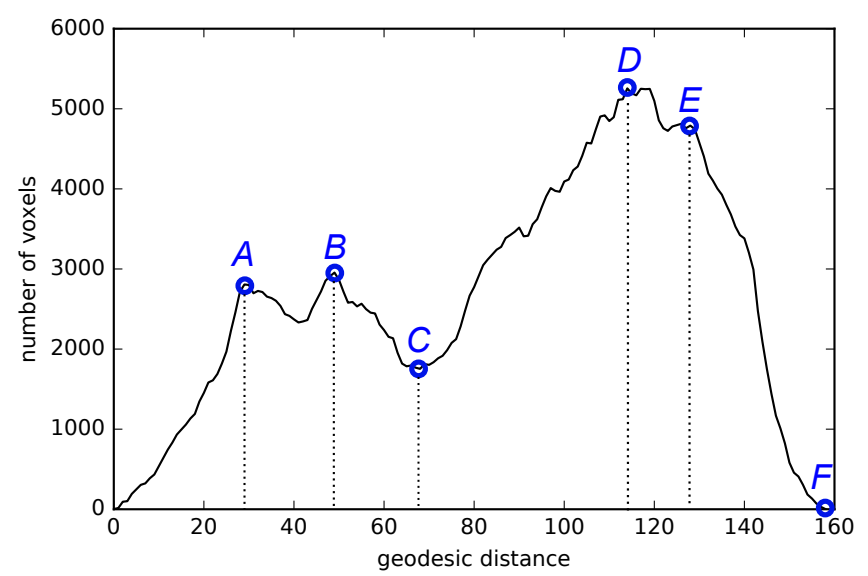

Figure 3: The line graph of the histogram of the geodesic distance map in Fig. 1 with some local extrema at $d=$ $29,49,68,114,126(A-E)$ and the rightmost $(F)$.

\subsection{Intrinsic profiling by geodesic propagation histogram}

We show the usefulness of the geodesic propagation histogram for intrinsic profiling of the intracranial cerebrospinal fluid within the subarachnoid space. As an example, the histogram of the geodesic distance map visualized in Fig. 1 is shown in Fig. 3.

We observe similarities between histograms of healthy adults: a bimodal shape with a first smaller peak. To understand such common points, we first chose the significant local extrema of the histogram. For example, the five extrema $(A-E)$ were selected at $d=29,49,68,114,126$ in Fig. 3. Then, we visualized in Fig. 4 the thresholded geodesic distance map with the distances of these extrema to observe the geodesic propagation evolution. In fact, the two-dimensional visualization using volumetric relief maps of the geodesic distance map with the same distance thresholds was also given in Fig. 2.

Figures 2 and 4 together with Fig. 3 allow us the following observations. The first peak mainly consists in the flooding of brain cisterns that surround the cerebellum and the brainstem as of spaces under the lateral cerebral fissure $(0-A-B-C)$. On the other hand, the second one corresponds to the flooding within the superior part of the subarachnoid space $(C-D-E-F)$.

These observations confirm that geodesic propagation histograms are useful to detect some critical distances where the manner of geodesic propagation evolution is changed and thus some local intrinsic structures would be modified as well.

\subsection{Bilateral intrinsic asymmetry detection}

To verify the bilateral intrinsic asymmetry, we made two geodesic propagation histograms for right and left hemispheres, $h_{r}$, and $h_{l}$, from a geodesic distance map. The reflection plane, i.e. the midsagittal plane was obtained as the bisector of the centers of the two eyeballs, which are easily extracted from original images as explained in [5]. A geodesic distance map is simply separated into two parts by this reflection plane, and a histogram is obtained for each one.

The two geodesic propagation histograms, $h_{r}$ and $h_{l}$, of the same healthy adult in Fig. 1 are shown in Fig. 5, together with the histogram for the entire sphere. The correlation between $h_{r}$ 


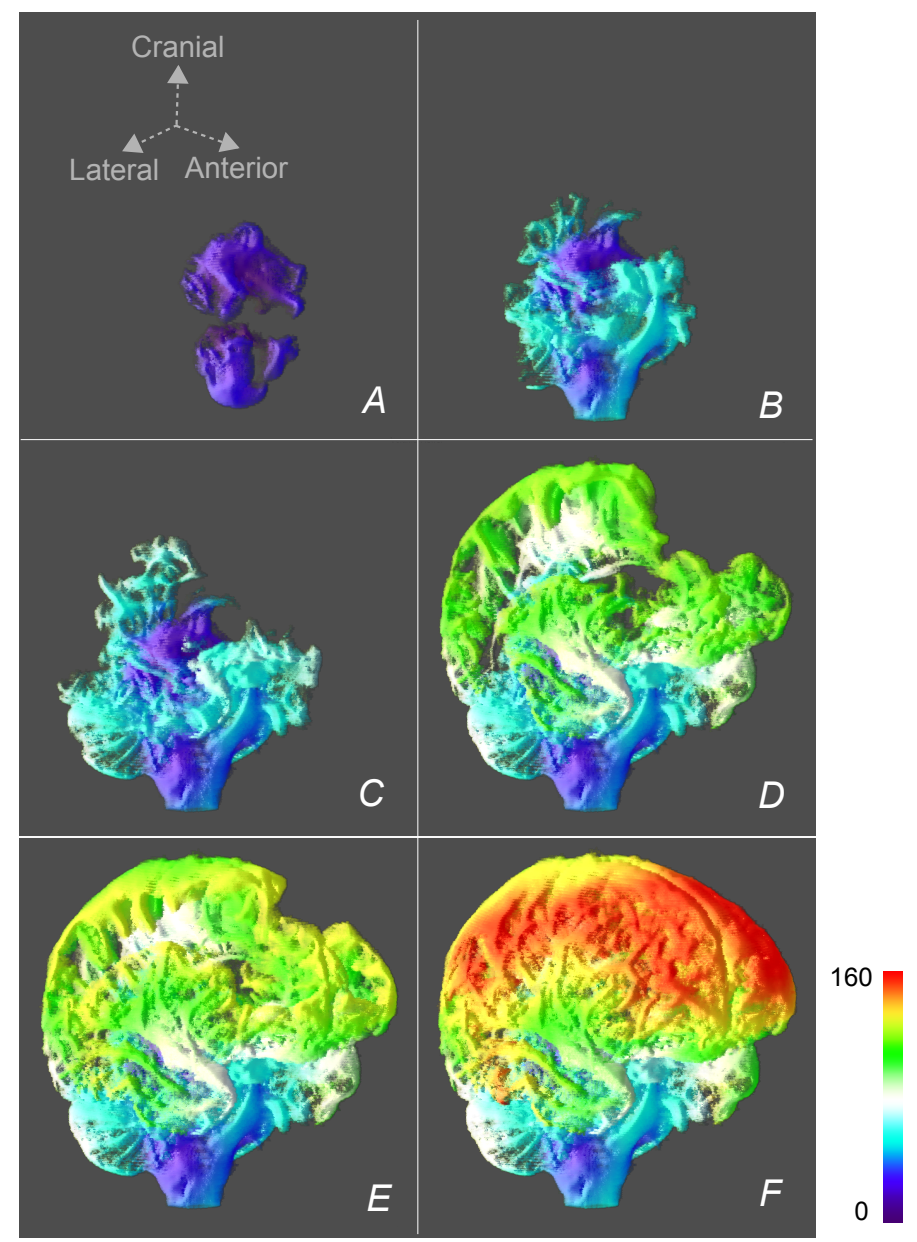

Figure 4: Colored visualization of the thresholded geodesic distance maps of with the different significant thresholds obtained from the geodesic propagation histogram in Fig. 3: $d=29(A), 49(B), 68(C), 114(D), 126(E)$ and $157(F)$. $(F)$ is a different view of the ones in Fig. 1. 


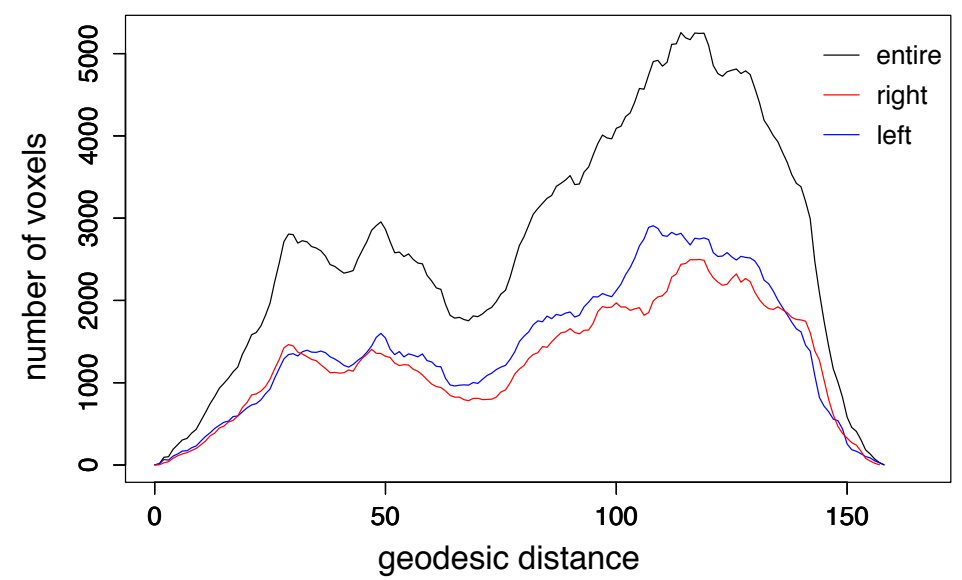

Figure 5: The two geodesic propagation histograms for the left and right hemispheres of the same healthy adult in Fig. 1 , together with the one for the entire sphere.

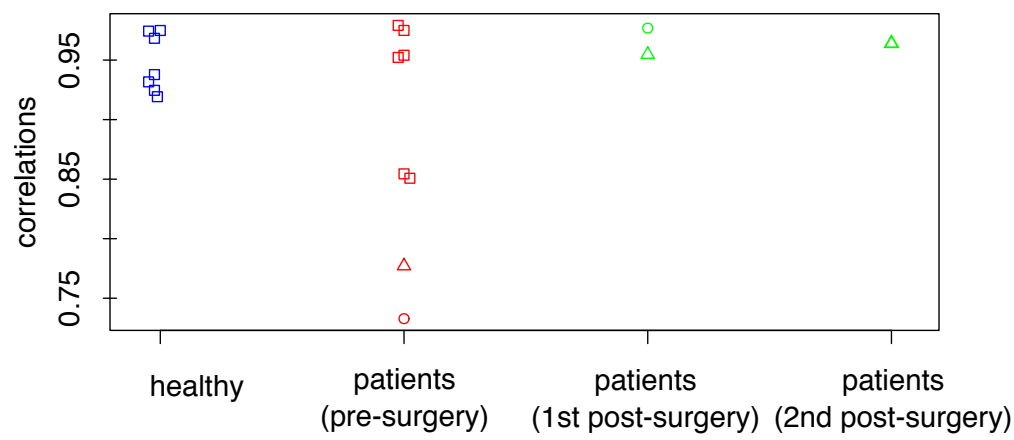

Figure 6: Distribution of correlations between left and right histograms $h_{r}$ and $h_{l}$ for 7 healthy adults and 8 patients, among which there are the ones of two patients (with the red circle and triangle) together with post-surgery histogram correlations (with the green circle and triangles (twice), respectively).

and $h_{l}$ is 0.9683 . As seen in Section 2.5, it can be said that the intracranial cerebrospinal fluid is not bilaterally (intrinsically) asymmetric if the correlation is close to 1 . Similar results are also obtained for 6 other healthy adults, that is, they are not bilaterally (intrinsically) asymmetric, as shown in Fig. 6.

The correlations between $h_{r}$ and $h_{l}$ are also calculated for 8 hydrocephalus patients, which are indicated in Fig. 6. Contrary to the results of the healthy adults, they are distributed in a wider range between 0.7 and 1.0. This signifies that some types of hydrocephalus would cause bilaterally intrinsic asymmetric fluid pathways of the intracranial cerebrospinal fluid.

Among them, we show an example of a hydrocephalus patient before and after surgery, whose geodesic propagation histograms are illustrated in Fig. 7. The correlations between $h_{r}$ and $h_{l}$ before and after surgery are 0.7328 and 0.9768 , respectively, marked by the red and green circles in Fig. 6. In other words, $h_{r}$ and $h_{l}$ mismatch before surgery while they become more similar after. Similar results are also obtained for another patient who had image acquisition twice after surgery, as shown using the red and two green triangles in Fig. 6 (the green ones correspond 

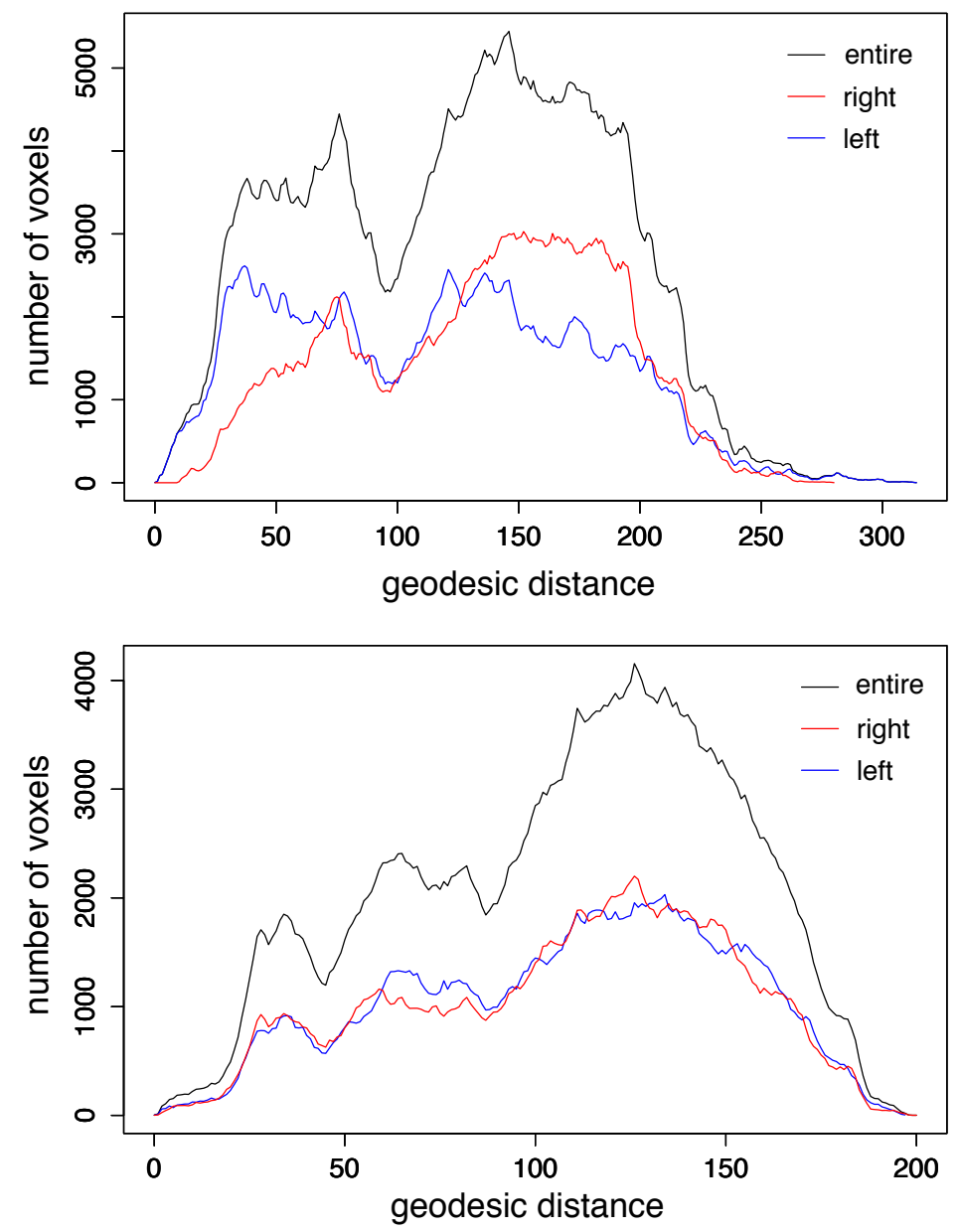

Figure 7: The two geodesic propagation histograms for the left and right hemispheres of a patient before and after surgery (top and bottom respectively), together with the histogram for the entire sphere. (See the attached mp4 files, video2 and video3, for the colored volumetric relief map sequences illustrating the geodesic distance map for the right hemisphere of the patient, before and after surgery.) 


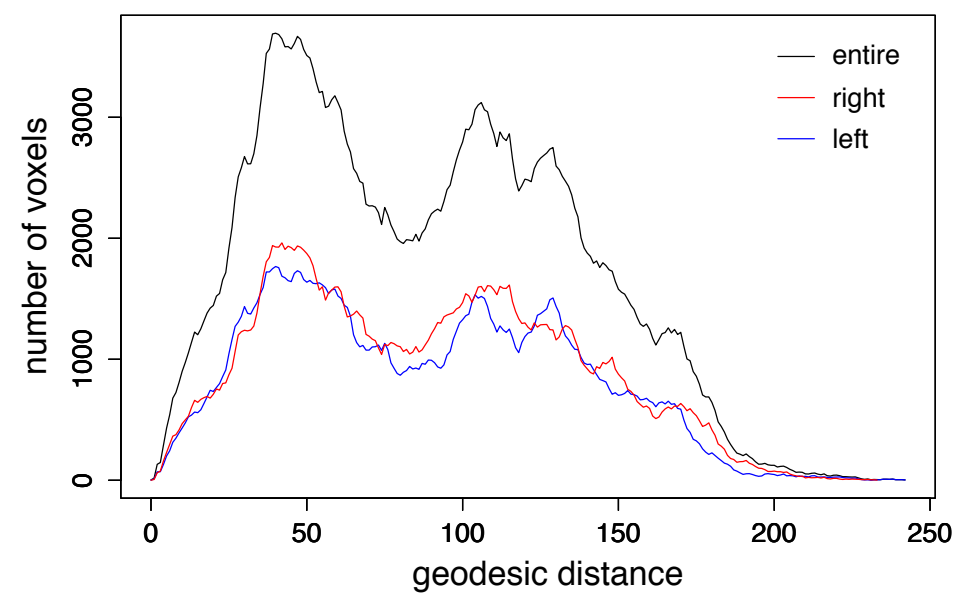

Figure 8: The two geodesic propagation histograms for the left and right hemispheres of a hydrocephlus patient, together with the one for the entire sphere.

to the first and second results after surgery, respectively). We thus can conclude that the intracranial subarachnoid space was bilaterally (intrinsically) asymmetric before surgery and such asymmetry has disappeared after surgery.

As shown in Fig. 6, the correlation between histograms $h_{r}$ and $h_{l}$ of a hydrocephalus patient is sometimes near 1. Figure 8 illustrates the histograms of the high-correlation case $(0.9749)$ observed as one of the healthy adults in Fig. 6. It signifies that the intrinsic profile greatly differs from the typical one of healthy adults as shown in Fig.3, even if the left and right histograms are highly correlated.

\subsection{Intrinsic profile changes in pre- and post-surgery}

Geodesic propagation histograms of a hydrocephalus patient pre- and post-surgery illustrated in Fig. 7, whose correlations between the left and right ones are shown as red and green circles in Fig. 6, also provide the information of intrinsic profile changes. Pre-surgery, we notice a substantial fluid accumulation in the inferior left hemisphere and one in the superior right hemisphere, which results in a significant bilateral asymmetry. Accumulation and asymmetry disappear postsurgery. It should also be noticed that the longest geodesic distance shrank post-surgery, while the total fluid volume, i.e., the total number of voxels, decreased, as already observed via volumetric relief maps in [6].

\subsection{Classification of intrinsic profiles}

To study intrinsic profile differences between healthy and pathological cases, we made the following classification into two classes using support vector machine. As mentioned in Section 2.6, here the 16-dimensional feature vector based on histogram moments of the four divided spherical parts, was utilized for each subject.

Obtaining the 16-dimensional feature vector requires the traverse plane as well as the midsagittal plane, which is already presented for the bilateral asymmetry detection in Section 3.3. The traverse plane was calculated as the plane that contains two eyeballs and the spherical center, which are easily chosen from the original image as explained in [5]. The obtained midsagittal 
and traverse planes have divided a geodesic distance map into four parts, and a histogram was obtained for each, whose moments were used as inputs for the classification.

In the experiment, the problem was treated as a two-class classification (healthy or not). We tested the model with a leave-one-out cross-validation, as the size of our dataset was limited. As the kernel function, we chose the radial basis function, one of the common kernels, and tuned the kernel parameter $\gamma$ and the cost of constraint violation, using a grid search, for each of leaveone-out setting from 14 training samples. The classification results were suitable; sensitivity, specificity and precision are all $100 \%$.

\section{Conclusion}

In this article, we investigated intrinsic structures of the intracranial subarachnoid space by observing the geodesic propagation from the specific fluid sources that correspond to the ventricular space outputs. We analyzed the propagation using a geodesic propagation histogram, which allows extraction of an intrinsic profile of the intracranial subarachnoid space, detection of its bilateral intrinsic asymmetry in pathological cases, and classification into two classes, healthy and pathological cases. The effectiveness of the proposed method was shown by the experiments on healthy adults and hydrocephalus patients. As the experiments were still preliminary, further work on statistical analysis with more data is expected, in particular, statistical shape analysis of geodesic propagation histograms.

\section{Acknowledgments}

The authors thank Jérôme Hodel, Pr. MD., Department of Neuroradiology, Hôpital Henri Mondor, Créteil, France, for the "fluid specific" MR image dataset used in this study.

\section{References}

[1] L. Sakka, G. Coll, J. Chazal, Anatomy and physiology of cerebrospinal fluid, Eur Ann Otorhinolaryngol Head Neck Dis 128 (6) (2011) 309-316.

[2] M. Matsumae, O. Sato, A. Hirayama, N. Hayashi, K. Takizawa, H. Atsumi, T. Sorimachi, Research into the physiology of cerebrospinal fluid reaches a new horizon: Intimate exchange between cerebrospinal fluid and interstitial fluid may contribute to maintenance of homeostasis in the central nervous system, Neurol Med Chir (Tokyo) 56 (2016) 416-441.

[3] R. Corns, A. Martin, Hydrocephalus, Surgery 30 (3) (2012) 142-148.

[4] C. Rosén, O. Hansson, K. Blennow, H. Zetterberg, Fluid biomarkers in alzheimer's disease - current concepts, Molecular Neurodegeneration 8 (1) (2013) 20.

[5] A. Lebret, J. Hodel, A. Rahmouni, P. Decq, E. Petit, Cerebrospinal fluid volume analysis for hydrocephalus diagnosis and clinical research, Comput Med Imag Grap 37 (3) (2013) 224-233.

[6] A. Lebret, Y. Kenmochi, J. Hodel, A. Rahmouni, P. Decq, E. Petit, Volumetric relief map for intracranial cerebrospinal fluid distribution analysis, Comput Med Imag Grap 44 (2015) 26-40.

[7] H. Ling, D. W. Jacobs, Shape classification using the inner-distance, IEEE Transactions on Pattern Analysis and Machine Intelligence 29 (2) (2007) 286-299.

[8] X. Bai, L. J. Latecki, Path similarity skeleton graph matching, IEEE Transactions on Pattern Analysis and Machine Intelligence 30 (7) (2008) 1282-1292.

[9] J. Rabin, G. Peyré, L. D. Cohen, Geodesic shapes and surfaces retrieval via optimal mass transport, in: Proc. of ECCV'10, Springer-Verlag, 2010, pp. 771-784.

[10] D. Raviv, A. Bronstein, M. Bronstein, R. Kimmel, Symmetries of non-rigid shapes, in: NRTL, 2007, pp. 1-7.

[11] C. P. Klingenberg, M. Barluenga, A. Meyer, Shape analysis of symmetric structures: quantifying variation among individuals and asymmetry, Evolution 56 (10) (2002) 1909-1920. 
[12] J. Hodel, J. Silvera, O. Bekaert, A. Rahmouni, S. Bastuji-Garin, A. Vignaud, E. Petit, B. Durning, P. Decq, Intracranial cerebrospinal fluid spaces imaging using a pulse-triggered three-dimensional turbo spin echo MR sequence with variable flip-angle distribution, Eur Radiol 21 (2) (2011) 402-410.

[13] J. A. Sethian, Level set methods and fast marching methods: Evolving interfaces in computational geometry, fluid mechanics, computer vision, and materials science, 2nd Edition, Cambridge University Press, Cambridge, 1999.

[14] T. H. Cormen, C. E. Leiserson, R. L. Rivest, C. Stein, Introduction to algorithms, 3rd Edition, MIT Press, Cambridge, MA, 2009

[15] P. Ngo, Y. Kenmochi, N. Passat, H. Talbot, Topology-preserving conditions for 2d digital images under rigid transformations, Journal of Mathematical Imaging and Vision 49 (2) (2014) 418 - 433.

[16] W. K. Pratt, Digital Image Processing, 4th Edition, John Wiley \& Sons, 2007.

[17] C. Cortes, V. Vapnik, Support-vector networks, Machine Learning 20 (3) (1995) 273-297. 